\title{
The Role of Videourodynamic Studies in Diagnosis and Treatment of Vesicoureteral Reflux
}

\author{
By R.J. Scholtmeijer and D.J. Griffiths \\ Rotterdam, The Netherlands
}

\begin{abstract}
- From January 1986 to January 1988, 63 children with 95 refluxing ureters have been studied in a prospective study with videourodynamic examination. All children with reflux grades I, II, and III received antibacterial treatment. Surgical treatment was adopted for reflux grades IV and V. provided detrusor instability had been excluded. However, if there was detrusor instability, anticholinergic drugs and antibacterial treatment were given in all grades of reflux and videourodynamic examination was repeated after 3 to 6 months and after 12 months of therapy. Bllateral reflux was found in 22 of 38 patients with a stable bladder, and reimplantation was performed in 11 patients with 18 refluxing ureters. In $\mathbf{2 5}$ children with 35 refluxing ureters of various grades of reflux, detrusor instability was found and unilateral reflux was noted more frequently than bilateral reflux. Surgery was necessary for only five children, because in the majority of the patients detrusor instability and reflux could be treated by anticholinergic drugs and antibacterial treatment. For the decision as to which treatment should be given in vesicoureteral reflux, a videourodynamic study is mandatory.

(c) 1990 by W.B. Saunders Company.
\end{abstract}

INDEX WORDS: Vesicoureteral reflux, videourodynamic examination.

D URING THE LAST 30 ycars, an increasingly large volume of clinical and experimental data have been reported on the clinical management of vesicoureteral reflux. ${ }^{1}$ When vesicoureteral reflux in children was discovered through cystography it was believed that the surgical cure of reflux was a promising way of treating urinary tract infection and its complications. ${ }^{2}$ Therefore, reimplantation of refluxing ureters was considered to be the best form of treatment. However, in the last 15 years the management of vesicoureteral reflux has become more controversial and it became difficult to decide the best form of treatment.

In a retrospective study of the urodynamic findings in 458 children, reflux was found in 104 of them. ${ }^{3}$ Two different types of reflux/dysfunction complexes with contrasting urodynamic characteristics could be distinguished. One type included bladder instability with powerful voiding contractions of the bladder and reflux that frequently occurred on one side only. In the other type the bladder contracted poorly during voiding and overactivity of the urethral closure mechanism often was observed. The bladder was usually stable and reflux occurred frequently on both sides.

If reflux is viewed as a urodynamic abnormality, the static cystographic.concept of reflux becomes an unac- ceptable tool for determining the treatment of choice. For this reason we have started a prospective urodynamic study.

\section{MATERIALS AND METHODS}

Since January 1986 all children suspected of having vesicoureteral reflux have undergone a videourodynamic examination. The majority of the patients suffered from recurrent urinary tract infections and received antibacterial treatment during a period of at least 4 weeks preceding the videourodynamic study. Filling and voiding cystometry are performed in a standard way, while the urinary tract is visualized intermittently at every increase of bladder filling with $50 \mathrm{~mL}$, during an instable contraction of the detrusor, and during micturition, which is recorded on videotape. Bladder and detrusor instability definitions conform to the standards recommended by the International Continence Society. ${ }^{4}$

According to the results of an other prospective study, ${ }^{5}$ all children with reflux grades I, II, and III (grading according to the International Reflux Study Committee ${ }^{6}$ ) receive antibacterial treatment in the first instance. Surgical treatment is adopted for refluxes grades IV and V, provided bladder instability has been excluded. However, if there is bladder instability anticholinergic drugs and antibacterial treatment are given regardless of the grade of reflux and the videourodynamic examination is repeated after 3 to 6 months of therapy. In cases in which reflux grades IV and V persists, surgery is considered. Patients who are not treated by surgery will have a videourodynamic examination repeated at 1,3 , and 5 years.

\section{RESULTS}

Between January 1986 and January 1988, 63 children with 95 refluxing ureters were studied. As described in our retrospective study, ${ }^{3}$ unilateral reflux was more frequently noted in children with an unstable bladder $(15 / 25)$. In cases of a stable bladder, bilateral reflux occurred more frequently $(22 / 38)$. In $27 \mathrm{pa}$ tients with a stable bladder who had 42 refluxing ureters, antibacterial treatment was started. Eleven patients, who had stable bladders, with 18 refluxing ureters were treated by reimplantation and antibacterial treatment.

For the group treated with antibiotics only the follow-up period is too short to draw any conclusions. But in the group treated with surgery plus antibiotics

From the Department of Paediatric Urology, Erasmus University and Sophia Children's Hospital, Rotterdam, The Netherlands.

Date accepted: July 31, 1989.

Address reprint requests to Professor Dr R.J. Scholtmeijer, Sophia Children's Hospital, Gordelweg 160, 3038 GE Rotterdam. The Netherlands.

(c) 1990 by W.B. Saunders Company.

0022-3468/90/2506-0021\$03.00/0 
the results after 1 year are known. Reflux was cured in nine patients with 14 reimplanted ureters. In two patients with grade $V$ reflux (three ureters) and one patient with grade III reflux on one side, a persistent grade III reflux was found in all ureters.

Special interest was directed towards the patients with bladder instability. In this group of 25 children with 35 refluxing ureters of varying grades of reflux, treatment consisted of anticholinergic drugs (oxybutinin HCL or oxyphenomiumbromide) plus antibiotics. The videourodynamic examination was repeated after 3 to 6 months and again after 12 months of therapy. After 3 to 6 months of therapy, reimplantation of eight urcters was necessary in five patients, because of persisting urinary tract infections with increase of bilateral reflux grade II to grade III in one patient and persisting grade IV and grade $\mathrm{V}$ reflux in the others. In all eight ureters the reimplantation was successful. The results of nonsurgical treatment after 12 months of therapy in the other 20 children with 27 refluxing ureters are listed in Table 1.

Grade 1 reflux originally noted in one patient disappeared. In 13 ureters originally with grade II reflux, after 12 months of therapy, six ureters no longer refluxed, three ureters were grade $I$, and in four ureters the reflux was unchanged.

In eight ureters originally showing grade III reflux, the reflux had disappeared in three, decreased to grade I in one, to grade II in two, and was unchanged in one. In one ureter no reflux was noted during filling of the bladder, but during micturition reflux grade IV reflux was noted.

The five ureters that showed grade IV reflux originally showed no reflux in one ureter after the 6-month study. But after 12 months the reflux was grade III. In one ureter there was a decrease to grade I at 6 months and there was no detrusor instability. Therefore, the anticholinergic treatment was stopped, but at the 12-month study there was some instability and the reflux was again grade IV (Figs 1, 2, and 3). In this

$\begin{aligned} & \text { Table 1. Results of Nonsurgical Treatment After } 1 \text { Vear in } 20 \\
& \text { Patients With } 27 \text { Refluxing Ureters and Detrusor Instability }\end{aligned}$
\begin{tabular}{cccccccc} 
Grade & No. of Ureters & 0 & I & II & III & IV \\
\hline I & 1 & 1 & & & & \\
II & 13 & 6 & 3 & 4 & & \\
III & 8 & 3 & 1 & 2 & 1 & 1 \\
IV & 5 & & & & 2 & $3 \dagger$ \\
Total & 27 & 10 & 4 & 6 & 3 & 4 \\
\hline
\end{tabular}

"Reflux occurring only during micturition

tAt 6 months, grade I (1) and grade III (2) reflux.

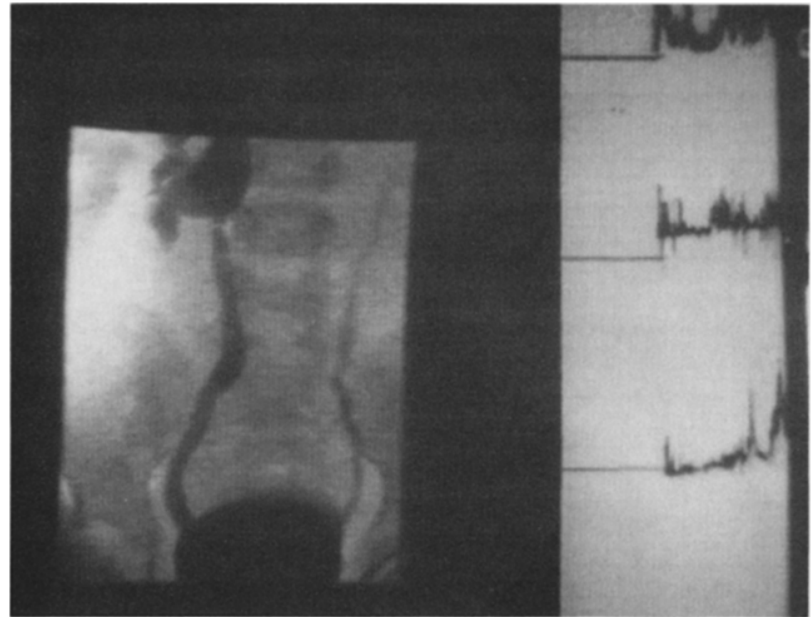

Fig 1. Fluoroscopy during an instable contraction of the $\mathrm{m}$. detrusor. (Right) Reflux grade IV; (left) grade II.

patient the anticholinergic treatment has been restarted.

In another ureter an initial decrease to grade II was noted, but there was an increase again to grade III at 12 months. In two ureters there was a decrease to grade III at 6 months, although no bladder instability was found. Therefore, the anticholinergic treatment was stopped, but in both the reflux was increased again to grade IV at 12 months and there was also a slight instability of the detrusor. In both patients the anticholinergic treatment has been restarted.

In all cases the bladder instability has been reduced or disappeared. However, after cessation of the anticholinergic therapy some degree of instability tend to recur, often associated with a resumption of the grade

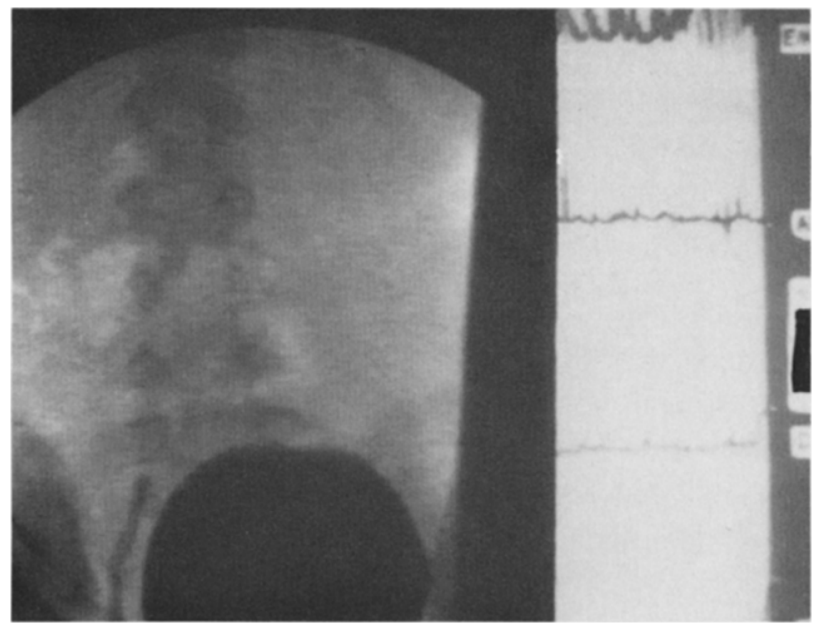

Fig 2. Videourodynamic study after 6 months of anticholinergic therapy. Stable bladder. (Right) Reflux grade I; (left) no reflux. 


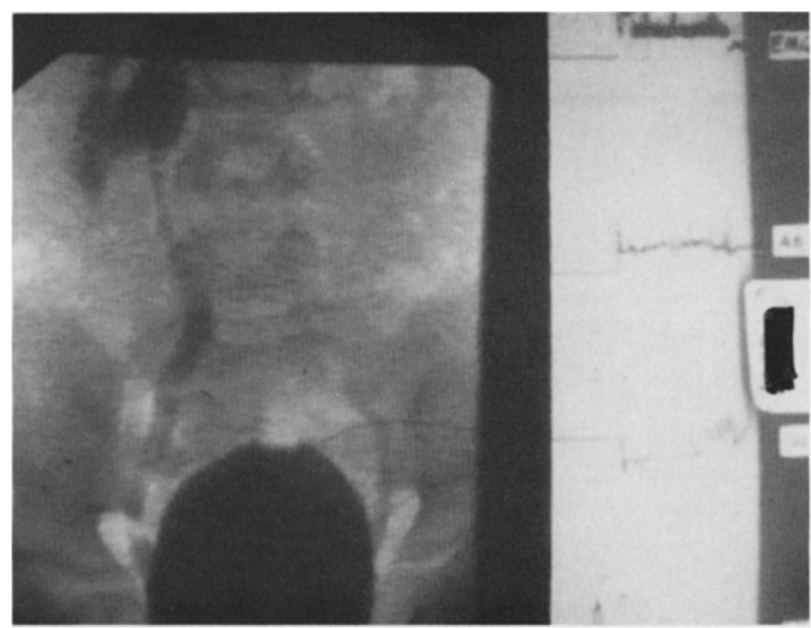

Fig 3. Videourodynamic study 6 months after cessation of treatment. Instable bladder again. (Right) Reflux grade IV; (left) reflux grade II.

of reflux that initially seemed to be decreased on therapy.

In patients with bladder instability the reflux disappeared in 10 of 27 refluxing ureters, whereas in eight other ureters a decrease to a lower degree of reflux was observed.

\section{DISCUSSION}

Until now the voiding-cystourethrogram has been the gold standard for detection and grading of vesicoureteral reflux. However, since 1983 attention has been directed towards vesicoureteral reflux in children with no overt abnormality other than urodynamically demonstrable dysfunction of the lower tract. Not only a congenitally abnormal implantation of the ureter into the bladder, but also urinary tract infections, bladder or urethral dysfunction with development of high bladder pressures, and ureteric peristaltic properties seem to be important in the development of reflux. ${ }^{3,7-9}$

In this prospective study with videourodynamic examination of all children suspected of having reflux, we found detrusor instability in 25 of 63 children (40\%). For only five of these children (20\%) with eight refluxing ureters, surgical treatment was necessary. This is in contrast to a group of 38 patients with stable bladders in which 11 patients $(29 \%)$ with 18 refluxing ureters were treated by reimplantation.

The results of the videourodynamic investigations confirm the findings of our retrospective study ${ }^{3}$ : vesicoureteral reflux is often caused by bladder/urethra dysfunction. Unilateral reflux is more often found in cases of bladder instability whereas in stable bladders reflux frequently occurs bilaterally. Moreover, in cases of bladder instability reflux can be cured or diminished within 6 to 12 months by anticholinergic drugs and antibacterial treatment, even in patients having grade IV reflux. Anticholinergic therapy reduces the need for surgical treatment.

Voiding cystourethrography is only suitable for detection of vesicoureteral reflux, but a videourodynamic study is suggested for the decision as to which treatment should be given.

\section{REFERENCES}

1. Bellinger MF: The management of vesicoureteric reflux. Urol Clin North Am 12:23-29, 1985

2. Bakker NJ, Griffiths DJ, Scholtmeijer RJ: The concept of vesicoureteral reflux. Urology 15:98-99, 1985

3. Griffiths DJ, Scholtmeijer RJ: Vesicoureteral reflux and lower urinary tract dysfunction: Evidence for two different reflux/ dysfunction complexes. J Urol 137:240-244, 1987

4. International Continence Society: Fourth report on the standarization of terminology of lower urinary tract function. $\mathrm{Br} \mathrm{J}$ Urol $51: 333-335,1981$

5. Scholtmeijer RJ, Griffiths DJ: Treatment of vesicoureteric reflux: Preliminary report of a prospective study. $\mathrm{Br} \mathrm{J}$ Urol 61:205209,1988

6. International Reflux Study Committee: Medical versus surgical treatment of primary vesicoureteral reflux: A prospective international study in children. J Urol 125:277-283, 1981

7. Nielsen JB, Djurhuus JC, Jörgensen TM: Lower urinary tract dysfunction in vesicoureteral reflux. Urol Int 39:29-31, 1984

8. Jörgensen TM: Pathogenic factors in vesico-ureteral reflux. Neurourol Urodyn 5:153-183, 1986

9. Koff SA, Murtagh DS: The uninhibited bladder in children: Effect of treatment on recurrence of urinary infection and on vesico-ureteral reflux resolution. J Urol 130:1138-1141, 1983 